\title{
Medical Devices based on substances
}

\author{
F. Gellona
}

Confindustria Medical Devices, Milan, Italy

The world of Medical Devices is not only a brand new and innovative sector but it is also a fascinating, very fascinating world, because every time we look back we see that it is putting us in situations that were not considered in the past or were considered in a different way. There is extraordinary value in this sector, the most suitable example of such value are Medical Devices made of substances (MDMS). Here I try to get to the core of this sector, its features, its opportunities and the challenges on which we have been working greatly with other associations in Europe and in Italy.

Italy, first of all the Ministry of Health (MoH), is highly qualified in the sector of Medical Devices and more specifically in MDMS.

Referring to the new regulation, we have to work with national and European institutions in order to stand up for ourselves and to ensure that the peculiarity of these products be considered.

We have been standing up to safeguard a growing market, because this means jobs, investments, wellbeing and appeal. At the same time, it is a sector that offers efficient therapeutic solutions and these new therapeutic solutions are very important. Therefore, I think it is a great opportunity for all EU citizens. I would like to give a glance to the industry of Medical Devices in Italy. We are talking about 5,000 companies, more than 76,000 employees, more than 11 billion turnover: it is a sector that has nothing to envy to other sectors that are more widely known, in the community, and are considered more precious. We also have other characteristics like a very high rate of education among our employees, given the sector. We have a strong presence of women. So again, as a sector, from this point of view, we try to be really at the forefront. Italy is the first European biomedical pole of manufacturing in the sector of Medical Devices.

Inside the diversified world of Medical Devices, we find those that are based on substances.

This kind of products is certainly one of the most important news in the European Regulation. They represent a new opportunity of care, that both medical and technological sciences are providing to be available to people.

Studies done in the last years demonstrate that these are not drugs, even when they have the same intended use. They are with full right Medical Devices.

How is it possible? Because they have different mechanisms of action from those required for drugs (the pharmacological mode of action). Companies invested and plan to increase the investments in specific studies to demonstrate this situation. We need to have a case by case approach based on experimental evidence.

At present, more than 4,000 companies are operating in the supply chain of medical devices. In MDMS we have 600 companies for a market that is worth more than 950 million Euros, with a very important market share accounting for $28 \%$. The pieces sold per year are 75.4 millions, accounting for $22 \%$ of the overall market share for this kind of use and purposes. We point out that also the big pharmaceutical companies are starting to be 
present in the market of Medical Devices made of substances. So, if we analyse this market, we see that it is constantly growing. We can see how the potential of MDMS has increased compared to OTCs. This sector is expanding constantly.

It is a constantly evolving market moving from 331 million Euros in 2010, to about more than 950 million in 2019.

The number of references that have been classified as Medical Devices is increasing, it has more than doubled over these years. From 1,002 references in 2010, we went to 2,620 only in 2019 (1). About 190 new references were launched as Medical Devices in 2019 (1). Therefore, this is an opportunity for innovation. Brands present in our everyday life, are now drawing the attention of the biggest pharmaceutical multi-nationals, not only the Italian ones, but also the foreign ones.

We took a specific case, the cough case in Italy, to show how the market has grown in Italy. In 2011, 66\% were mucolytic drugs, and $27 \%$ were sedative drugs (3), while the Medical Device was just a small residual share, accounting for $7 \%$.

In 2019, 18 years later, the share of Medical Devices grew up to $24 \%$. The cough case is a quite relevant and important case study on the potential of medical devices for therapeutic innovation. So what does it mean? It means that there is a strong expansion going on, 80 references in 2019, 25 launched in the last year. As we hinted above, all the main multi-nationals have penetrated this market. These companies, many of which foreign, have here in Italy an important market share for these MDMS. This means that our country is open to this kind of innovation. Some products can be traded here if they do not find a "mentally" open door in the country of origin.
This is a positive trend. There is sound competition here, good competition, that should not be hindered by legislative prohibitions that might somehow impair the dissemination of these products. From this point of view once again, the choice of physicians on the one hand, but most of all, of the patients, is self-evident. This is true also about other kinds of Medical Devices. If the final user, the citizen, the patient, can choose, that is sound competition. The patient knows whether that substance, that Medical Device, is working or not. If it has a certain level of efficacy, he or she is going to buy the product. It is self-evident. Industry investing, it is creating job opportunities, finally, it is creating culture. I think that in the world of Medical Devices, being that they originate from a combination of knowledge of every kind, a world of culture, science, knowledge, is extremely important. So once again, our Association supports strict regulations, but these regulations have to be suitable. Let us welcome a responsive market that welcomes innovation because that is the actual core mission of this industry and its products. So let us support this therapeutic, industrial and cultural trend, which is thankfully growing. Therefore, in a moment in which we have to provide answers to many health needs and wellbeing needs, we have to give a safe answer in terms of products, a suitable answer in terms of distribution, and quality. I think that the Italian market is at the forefront in this regard, and we hope to keep on with this performance.

\section{References}

1. IMS Health, Settembre 2010-2019 OTC/SOP and MDs.

2. IQVIA, Multichannel Italy, OTC/SOP and MDs - Sell out Data Mat Sept 2019. 